\title{
Quelles ressources et collaborations pour faciliter la scolarisation inclusive dans les établissements scolaires du $2^{\mathrm{e}}$ degré
}

Auteur

Patrice Bourdon, Maître de conférences - Ph. D. Sciences de l'éducation Université de Nantes/ESPE - CREN EA 2661, France,

patrice.bourdon@univ-nantes.fr 


\section{REVUE HYBRIDE DE L'ÉDUCATION}

\section{Résumé}

Cet article analyse le fonctionnement d'un lycée ( $2^{\mathrm{e}}$ degré) de Nantes (France) dans lequel différents partenaires collaborent depuis plus de 25 ans, dans un partenariat entre l'Éducation Nationale, une association pour adultes et jeunes handicapés (APAJH) et les collectivités territoriales (département et région). Une cinquantaine d'élèves en situation de handicap sont scolarisés dans les classes ordinaires avec un très bon taux de réussite aux examens. II a paru nécessaire d'investiguer le rapport entre les conditions spécifiques de fonctionnement de ce dispositif inclusif et la réussite scolaire des élèves, notamment sur les questions de collaboration et de coopération entre les différents professionnels afin d'identifier quelles sont les ressources mises en œuvre. II s'agit aussi d'identifier comment les partenaires s'en saisissent pour développer et consolider leurs activités au profit de l'inclusion. Nous mettons en évidence plusieurs fonctions ressources régulatrices de l'activité des professionnels.

Mots-clés: inclusion scolaire, collaboration, coopération, scolarisation inclusive, situation de handicap 


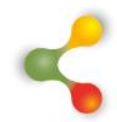

\section{REVUE HYBRIDE DE L'ÉDUCATION}

\section{Introduction}

L'article est issu des résultats d'une recherche que nous avons conduite en 2015 dans un lycée ${ }^{1}$ de Nantes en France et qui a donné lieu à publications (Bourdon et Toullec-Théry, 2016, 2018). Ici, il ne s'agit donc pas de se saisir à nouveau des résultats déjà publiés, mais d'explorer une partie de nos travaux qui a peu ou pas été diffusée. En effet, les enseignants ressources tels qu'ils sont encadrés de façon législative et tels qu'ils sont déployés au Québec dans les pratiques quotidiennes des établissements du $2 \mathrm{~d}$ degré, ne sont pas institutionnalisés de la même façon en France. En effet, jusqu'à la modification de la certification d'enseignant spécialisé par le décret $N^{\circ}$ 2017-169 du 10 février 2017 (CAPPEI2), la notion de «ressources » n'était pas identifiée en France comme étant une pratique spécifique en appui à la scolarisation inclusive. À ma connaissance, seul un article de paru dans les cahiers pédagogiques (Bourdon, 2008) avait abordé cette question mettant en articulation la fonction de spécialiste de certains enseignants versus enseignants ressources pour avancer vers une école inclusive. Dans cette réforme de 2017, il s'agit selon le texte « d'amener les enseignants à appréhender trois nouvelles dimensions du métier d'enseignant spécialisé : sensibiliser à l'éducation inclusive, jouer un rôle moteur dans l'environnement, apporter une expertise à l'équipe pédagogique ${ }^{3}$. Alors qu'au Québec, certains enseignants sont missionnés et identifiés comme "enseignantsressources " et que la tâche d'enseignant-ressource est régie par la convention collective des enseignants et devrait respecter les énoncés de l'annexe IV du référentiel sur les élèves à risque ou HDAA produit par la $\mathrm{FSE}^{4}$. En France, il s'agit plus d'un certain nombre de ressources dont les acteurs se saisissent sans que cela ne relève d'une mission dédiée à certains enseignants. Pour autant, parmi les fonctions d'enseignants spécialisés, nous l'avons vu ci-dessus, ils ont bien à construire des compétences pour être «ressources » de l'École inclusive. II s'agit donc plus d'un système de ressources sur lequel chacun peut s'appuyer pour réguler la scolarisation inclusive et résoudre les problèmes rencontrés au fil de l'activité.

Au lycée Les Bourdonnières, sur près de 180 professeurs, seuls trois sont spécialisés; la fonction "ressources » et la réponse à des besoins spécifiques ne peuvent donc pas uniquement passer par les

\footnotetext{
1 Le lycée correspond à une scolarité située entre 15 et 17 ans et donne lieu aux termes des trois années à un diplôme appelé « baccalauréat » qui permet l'accès à l'université

2 Certificat d'aptitude professionnelle aux pratiques de l'Education inclusive https://www.education.gouv.fr/bo/17/Hebdo7/MENE1704263C.htm?cid_bo=113028

3 https://www.education.gouv.fr/pid285/bulletin_officiel.html?cid_bo=113028 (annexe III-1-f)

4 http://3symposium.ctreq.qc.ca/wp-content/uploads/2016/05/La-fonction-denseignantressource-au-secondaire.pdf
} 


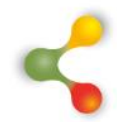

\section{REVUE HYBRIDE DE L'ÉDUCATION}

enseignants spécialisés. II existe par contre un dispositif appelé « centre de scolarité adaptée » (CSA) composé de différents personnels (enseignantcoordonnateur, auxiliaires de vie scolaire, personnels de soins et de rééducations, psychologue, etc.) qui apportent leurs expertises, sensibilisent les membres de la communauté éducative sur l'éducation inclusive et jouent un rôle moteur en appui aux équipes pour favoriser une scolarisation en milieu ordinaire d'élèves en situation de handicap. Comment alors se régule et s'organise la réponse aux besoins des élèves et surtout, dans quelle mesure les enseignants réguliers et les autres personnels du lycée (administration, agents de services, personnels de la vie scolaire, etc.) trouvent-ils des ressources pour répondre au mieux aux besoins éducatifs particuliers des élèves en situation de handicap ? Nous faisons l'hypothèse que le dispositif d'appui à la scolarisation inclusive permet un fonctionnement à partir des ressources produites par le CSA. Ce ne serait finalement pas un personnel particulier qui agirait en tant que ressources, mais un dispositif d'appui à la scolarisation des élèves en situation de handicap qui fonctionne et se régule au travers diverses fonctions que l'on peut qualifier de « ressources ", car elles favorisent une scolarisation inclusive. II n'y a pas, à proprement dit, d'enseignantressource dans cet établissement, mais un ensemble de professionnels ressources. II ne s'agit donc pas, par exemple, de pratiques proches de la co-intervention ou du coenseignement (Tremblay, 2015) telles qu'elles sont mises en œuvre au Québec. Certains enseignants.es sont « ressources » même s'ils ne sont pas nommés.es comme tels.les. Certains fonctionnements le sont aussi, certaines pratiques encore.

Ce qui nous intéresse donc dans cet article, c'est d'analyser les différentes fonctions qui font «ressources» et les collaborations conséquences mises en œuvre en appui de la scolarisation pour un public à besoins éducatifs particuliers.

Dans une première partie, nous présenterons le cadre de cette recherche puis nous développerons une sorte de modélisation des fonctions et leurs effets. Cela nous permettra également de mettre au jour les différents professionnels qui s'en emparent et vers qui ces actions viennent en appui dans leurs pratiques quotidiennes.

\section{Contexte de la recherche et cadre théorique}

Cette recherche peut être considérée comme une étude de cas (Passeron et Revel, 2005), qui tente de mettre en relation les éléments disjoints d'une configuration (ici le dispositif du lycée Les Bourdonnières), qui est, au départ, difficilement compréhensible dans sa globalité et sa complexité, y compris pour les acteurs eux-mêmes et qui pour cela, fait problème. Cette recherche nécessite alors une incursion dans différents registres (dans différents types de données). C'est l'ensemble de traces, d'indices recueillis qui va établir un réseau de significations et finir par faire signe (c'est-à-dire signifier quelque chose). Le cas est donc fondé sur 


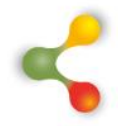

\section{REVUE HYBRIDE DE L'ÉDUCATION}

«l'approfondissement des singularités» via l'observation des "opérations" (de ce qui se passe) et non sur la généralisation par extension. Nous procédons par une description à partir de l'observation et l'expérience des acteurs du dispositif pour aboutir à une trame interprétative et à l'élaboration d'hypothèses explicatives.

Cette recherche s'ancre dans nos travaux antérieurs sur la scolarisation inclusive des élèves en situation de handicap (Bourdon, 2007, 2008 ; Bourdon et Toullec-Théry, 2016), mais aussi sur l'accompagnement des AVS, leur articulation avec les enseignants et les effets sur les apprentissages des élèves (Toullec-Théry, 2013). Nous mettrons à l'épreuve certains de nos résultats en les confrontant à un nouveau milieu, ce lycée qui privilégie ouvertement l'inclusion et lui attribue des moyens en espace, temps et personnel. L'enjeu est donc d'étudier un système complexe, c'est-à-dire, au sens de Saussure :, " un ensemble organisé d'éléments indissociables qui interagissent [...], dépendants les uns des autres pour former un tout organisé » (De Rosnay, 1975, p92), système qui fonctionne "en interaction dynamique organisé en fonction d'un but» (Ibid., p101). Nous avons tenté de croiser deux cadres théoriques : celui de la théorie de l'activité dans le sillage de Leontiev (1975) ou de Rochex (1995) et la Théorie de l'Action Conjointe en Didactique développée par Sensevy (Sensevy et Mercier, 2007). II s'agit ici d'expliquer la mobilisation des acteurs et la façon dont ils construisent leurs activités, tout en focalisant notre attention sur les objets sur lesquels les acteurs portent leur attention. Accordent-ils une place prégnante aux savoirs et connaissances ou privilégient-ils les organisations? Nous savons en effet aujourd'hui que la forme scolaire détermine les modalités d'organisation des pratiques et par là même, les pratiques enseignantes.

Pour cette recherche, nous avons recueilli le discours d'une grande partie des acteurs impliqués dans le dispositif au cours d'entretiens enregistrés. Ils sont de deux types : des entretiens semi-directifs centrés (Blanchet et Gotman, 1992) et des instructions au sosie (Clot, 1995). Ces données, intégralement transcrites sont ensuite analysées selon un processus d'enquête (synopsis, narrations, typologies) et nous permettent de construire progressivement un réseau de significations. Nous avons par ailleurs assisté à trois réunions d'AESH ${ }^{5}$ qui ont été enregistrées et analysées. Le public cible des entretiens est ainsi composé :

- des cadres dirigeants d'une association partenaire: APAJ6 (président et vice-présidente),

- du proviseur (directeur) du lycée,

5 Accompagnant d'élève en situation de handicap (AESH) appelé aussi auxiliaire de vie scolaire (AVS)

6 Association pour jeunes et adultes en situation de handicap (https://www.apajh44.org/fr/) 


\section{$\&$}

\section{REVUE HYBRIDE DE L'ÉDUCATION}

- d'un coordonnateur du $\mathrm{CSA}^{7}$ et de la coordonnatrice des AESH,

- d'un psychologue et de rééducateurs (orthophoniste et kinésithérapeute),

- des AESH,

- de trois professeurs (P1- P2 et P3),

- de quatre élèves.

Nous avons ainsi mené 10 entretiens de professionnel (cinq instructions au sosie et cinq entretiens semi-directifs) et enregistré trois réunions de travail des $\mathrm{AESH}$. L'objectif était de recueillir des traces de l'activité à travers les discours produits, mais aussi d'identifier les freins et facilitateurs qui apparaissent lors de ces temps de concertation. Les corpus sont analysés dans leur intégralité à partir des discours produits en lien avec les contenus. Aussi, nous pouvons considérer qu'il s'agit d'une analyse des pratiques langagières (Bautier, 1995).

\section{Une infrastructure et des personnels qui répondent aux besoins}

Le Lycée Les Bourdonnières à Nantes est un lycée général, technologique et professionnel qui accueille, généralement sur trois années, environ 2000 élèves de la seconde à la terminale (année de diplomation). II a été ouvert en 1966 et le dispositif d'accompagnement à la scolarisation des élèves en situation de handicap a quant à lui démarré en 1987.

170 professeur.es exercent dans cet établissement dont quatre qui ont suivi une formation d'enseignant.es spécialisé.es. Les élèves peuvent choisir un parcours de formation dans une des séries économique et sociale, littéraire ou scientifique correspondant à la voie générale ou dans un des domaines du transport, de la logistique, du tertiaire et du sanitaire, et social, correspondant aux voies technologiques et professionnelles, ${ }^{8}$

précise le site internet du lycée. Concernant le public à besoins éducatifs particuliers, ce sont une soixantaine de lycéens avec des troubles moteurs pour la majorité, des troubles sensoriels (auditifs ou visuels) en minorité, quelques rares situations d'élèves avec autisme ou quelques élèves avec des troubles de la sphère "dys ». L'établissement dispose d'une quinzaine d'auxiliaires de vie scolaire mutualisés ${ }^{9}$ qui accompagnent les élèves en

7 Centre de scolarité adaptée

8 https://bourdonnieres.paysdelaloire.e-lyco.fr/etablissement/

9 Nous appelons «mutualisés " des accompagnants aux élèves en situation de handicap (AESH) dont les missions s'adressent à l'ensemble de la population scolaire à BEP et non 


\section{$\&$}

\section{REVUE HYBRIDE DE L'ÉDUCATION}

situation de handicap dans leurs différents moments de vie (en classe, en études accompagnées, lors de la restauration scolaire ou à l'internat).

Par ailleurs, et c'est là l'originalité de l'établissement, un centre de scolarité adaptée (CSA) est installé dans le lycée, véritable dispositif d'appui à la scolarisation des élèves à besoins éducatifs particuliers (BEP). Il est géré conjointement par l'éducation nationale et une association ${ }^{10}$ dite du " médico-social », qui apportent du personnel spécifique (kinésithérapeute, ergothérapeute, éducateur, psychologues, médecins, etc.) ainsi qu'une prise en charge des matériels et locaux dédiés aux soins. Enfin, l'internat adapté de huit places qui jouxte le CSA au rez-de-chaussée de l'internat classique, permet le pensionnat d'élèves dont le domicile est éloigné, comme pour une partie des élèves de ce lycée, mais qui comporte un accès au bâti, des infrastructures spécifiques, du mobilier et des sanitaires adaptés ainsi que du personnel d'accompagnement. Le CSA dispose également d'un certain nombre d'outils d'adaptation des supports pédagogiques (transcripteur braille, loupe ordinateur, photocopieuse dédiée à l'agrandissement ou la reproduction de documents spécifiques, salles d'études avec un.e AESH, etc.).

On peut donc opérer une première modélisation figurative pour mettre en lumière les différents éléments "ressources » en eux-mêmes, bien avant les pratiques effectives, pour identifier ce qui favorise une scolarisation inclusive.

uniquement à un élève dédié comme c'est parfois le cas pour les AESH individualisés. Nous reviendrons sur cette organisation et ses effets.

10 Ici l'APAJH : le médico-social regroupe les activités des établissement spécialisés pour enfants, adolescents ou adultes en situation de handicap. Ils sont généralement associatifs. 


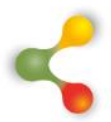

\section{REVUE HYBRIDE DE L'ÉDUCATION}

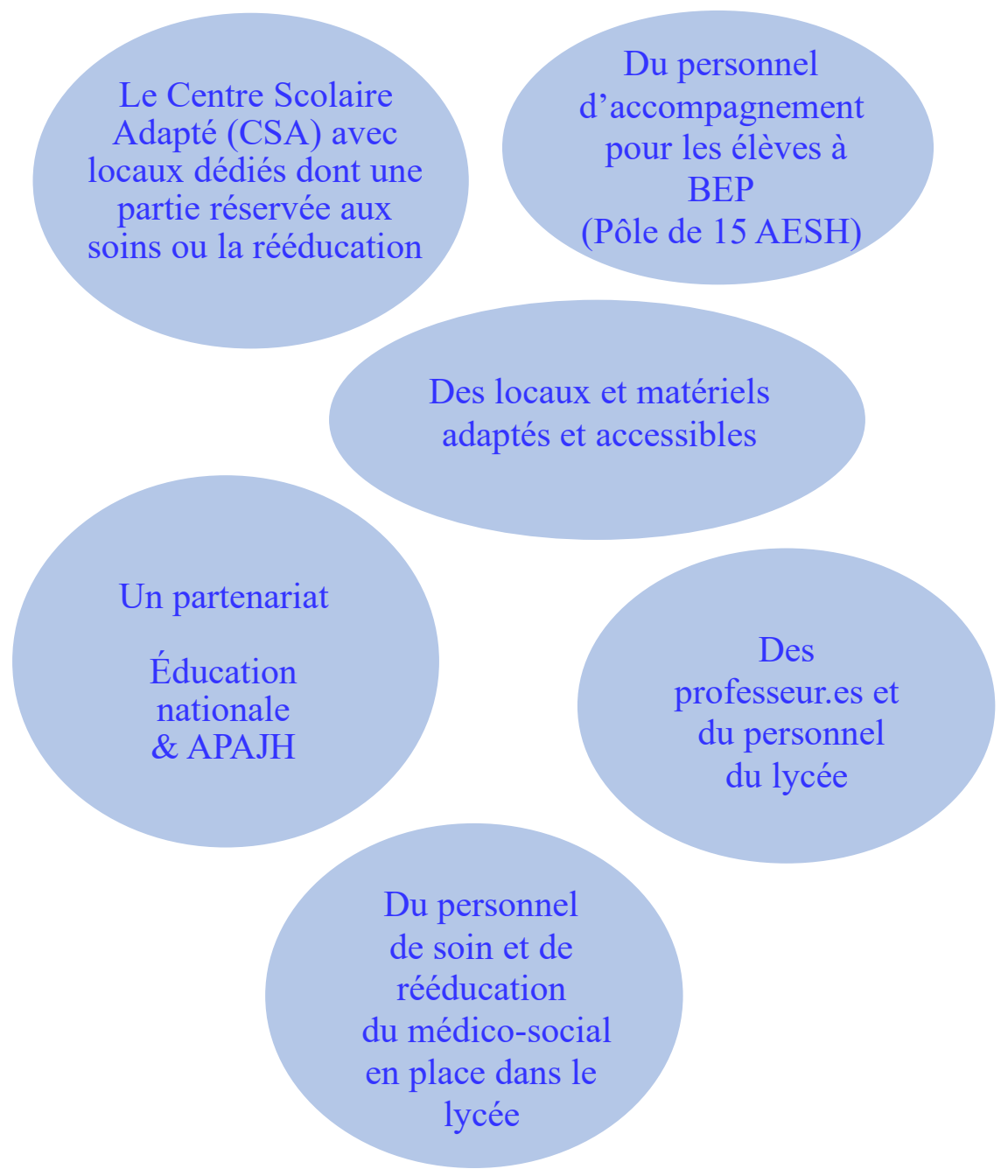

Figure 1. Modélisation des différents « pôles » de ressources

Un public à besoins éducatifs particuliers... comme les autres

La première question que l'on peut poser est de savoir quelle est la spécificité de ce public tant dans leurs besoins que dans l'organisation de leur scolarité. Les élèves qui bénéficient de ce dispositif sont-ils des élèves comme les autres dans leur rapport aux savoirs et à l'école, et dans le traitement qui est fait de la prise en compte de leurs besoins? Cette question peut paraître étonnante, voire saugrenue. Pourtant, elle est à 


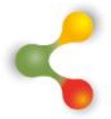

\section{REVUE HYBRIDE DE L'ÉDUCATION}

l'origine de notre recherche. En effet, il existe de nombreux dispositifs d'appui à la scolarité inclusive, depuis de nombreuses années, dans la plupart des pays, à des degrés divers de qualité. C'est le cas aussi en France puisque les premières lois sur l'intégration scolaire datent de 1975, suivies depuis par de nombreuses circulaires ou des cadres institutionnels : des dispositifs pour venir en appui à cette scolarisation qui requiert généralement des moyens particuliers ${ }^{11}$. Dans ce cas précis, ce qui a attiré notre attention fut lié au taux de réussite aux examens de ces élèves qui peinent parfois à maintenir leur scolarité, à valider des diplômes compte tenu à la fois des problématiques personnelles qu'ils rencontrent et des cadres de scolarisation qui leur sont proposés. En effet, dans ce lycée, les taux de réussite au baccalauréat sont semblables à leurs pairs (sans besoins éducatifs particuliers), que ce soit par l'obtention du baccalauréat ou par le pourcentage de mentions honorifiques obtenues reflétant la moyenne générale aux examens.

Par exemple, en 2013, 18 candidats se présentent au baccalauréat, 16 y sont admis, dont six qui obtiennent une mention, soit une moyenne de $89 \%$ de réussite, dont $33 \%$ avec mention. Deux d'entre eux échouent et redoublent. Si l'on rapproche les résultats spécifiques de ces élèves en situation de handicap avec ceux du lycée, le taux de réussite au baccalauréat est situé entre 89 et $91 \%$ ces trois dernières années; il est donc indéniable que les élèves en situation de handicap réussissent aussi bien que les autres.

Lorsque l'on examine le nombre moyen d'années pour obtenir ce diplôme, on s'aperçoit que les élèves en situation de handicap réussissent leurs examens en restant au lycée en moyenne six mois de plus que les autres élèves. II existe en effet une possibilité offerte a priori d'étalement de la scolarité en quatre ans, au lieu de trois, après validation en équipe de suivi de la scolarité (ESS). Cette possibilité est envisagée comme une réponse aux besoins de l'élève, par exemple pour des suites opératoires ou une fatigabilité importante. II ne s'agit pas d'un redoublement au sens habituel, mais bien d'une scolarité sur un an et demi ou deux ans avec étalement des contenus et des évaluations.

II nous faut donc préciser quelles sont les conditions d'admission aux études pour cet établissement. Ainsi, comme pour tous les élèves, ces derniers arrivent au lycée Les Bourdonnières par orientation du conseil de classe $^{12}$ de l'année précédente. C'est-à-dire qu'ils sont scolarisés dans le

11 Voici par exemple deux dispositifs significatifs en France : ULIS : Unité localisée pour l'inclusion scolaire - https://eduscol.education.fr/cid53163/les-unites-localisees-pour-Iinclusion-scolaire-ulis.html PIAL: Pôle inclusif d'accompagnement localisé https://www.education.gouv.fr/cid142647/ecole-inclusive-le-pial-qu-est-ce-que-c-est.html

12 Le conseil de classe est chargé du suivi des élèves et des questions pédagogiques intéressant la vie de la classe. Composé de membres du personnel de l'établissement, de délégués d'élèves et de parents d'élèves, il se réunit au moins trois fois par an. II formule des 


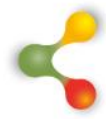

\section{REVUE HYBRIDE DE L'ÉDUCATION}

lycée non pas parce qu'ils sont en situation de handicap, mais parce que leur niveau scolaire le permet. "Leur admission, leur orientation scolaire, les enseignements suivent les mêmes procédures que pour tout autre élève. Ils peuvent bénéficier parallèlement d'une prise en charge médicosociale sur décision de la Maison Départementale des Personnes Handicapées (MDPH) »13, précise la plaquette du lycée. Ils disposent donc potentiellement d'un niveau de compétences et de connaissances validé par l'institution scolaire qui leur permet d'honorer les apprentissages au même titre que les élèves sans BEP. Pour autant, et nous y reviendrons, dans la mesure où une de nos hypothèses réside dans le fait que les compensations et l'accessibilité proposées dans cet établissement aident les élèves à progresser dans leurs apprentissages, il est fort à parier que sans le centre de scolarité adaptée et la culture spécifique du lycée autour du handicap (Bourdon, Toullec-Théry, 2016), le taux de réussite serait moins élevé. En d'autres termes, quelles sont les ressources mobilisées pour favoriser une scolarisation inclusive visant la réussite aux examens et une formation donnant accès à l'enseignement supérieur pour ceux qui le souhaitent?

Ce public a besoin de soins réguliers dans la semaine, voire la journée. Alors qu'habituellement ceux-ci sont pratiqués dans le cadre familial ou en "exfiltrant» l'élève de la classe pour le conduire dans un centre de soins externalisés, ici, les rendez-vous sont organisés dans l'établissement durant le temps scolaire, lors des pauses ou heures libérées entre deux cours. Nous avons montré dans nos travaux combien les élèves à BEP avaient affaire, plus que les autres, à des transitions nombreuses faites de ruptures et de continuités durant leur scolarisation (Bourdon, 2016, 2018 et 2019). Certaines de ces transitions sont subies, anticipées ou non, choisies, liées à des aléas de parcours, à une organisation spécifique et agissent pour colorer le mode de scolarité. C'està-dire qu'a priori, ce fonctionnement, qui permet une localisation des soins dans un contexte d'établissement scolaire durant la journée de classe et intégré à l'emploi du temps scolaire, va favoriser la continuité du parcours d'élèves en leur évitant de jongler entre les cours au lycée, les soins en dehors de l'établissement et la nécessité de "rattraper" ce qui a été manqué en enseignement compte tenu des trajets et de l'éloignement des centres de soins. C'est donc une organisation qui convient aux activités scolaires de l'élève et non le contraire qui serait un premier gage de parcours inclusif. La ressource est locale et non externalisée hors des lieux de vie quotidiens des élèves.

propositions concernant l'orientation et l'accompagnement des élèves et en informe les parents. https://www.service-public.fr/particuliers/vosdroits/F1394

13 https://bourdonnieres.paysdelaloire.e-lyco.fr/etablissement/centre-de-scolarite-adaptee/ 


\section{$\&$}

\section{REVUE HYBRIDE DE L'ÉDUCATION}

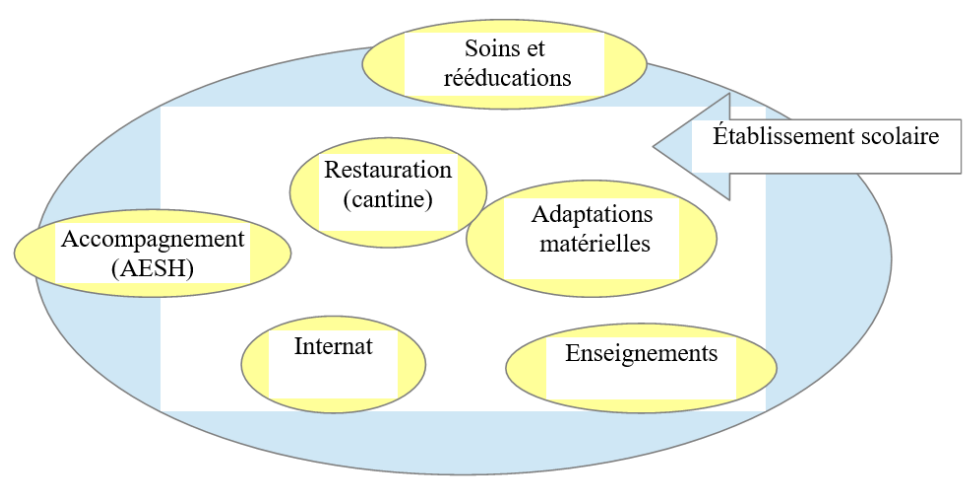

Figure 2. Modélisation des différents milieux d'activités

\section{Des ressources qui se concrétisent par des fonctions}

On peut en effet considérer que la "fonction ${ }^{14}$ tel que nous l'employons, relève à la fois de processus et de procédures, qu'elle engage des institutions et des acteurs (des professionnels et des politiques). On peut alors retenir la définition suivante de la fonction comme étant « une activité déterminée dévolue à un ensemble ou à un élément de l'ensemble ${ }^{15}$. C'est ainsi un élément qui assure une stabilité dans les théories fonctionnalistes, c'est à dire en référence à l'usage qu'un organe social (le centre scolaire adapté, par exemple) joue dans une organisation sociale donnée, pour la réguler. En ce sens, la fonction assure la stabilité d'une organisation. On peut aussi indiquer qu'elle se déploie dans une organisation hiérarchisée, d'une part parce le système qui est installé pour venir en appui de la scolarisation des élèves à BEP dans cet établissement est complexe. Elle est le fruit d'une histoire (depuis 1987), d'une géographie (les locaux, l'architecture, l'environnement, les transports) et d'une cause (l'inclusion) ${ }^{16}$. D'autre part, c'est une organisation dans un système composé de multiples professionnels n'appartenant pas aux mêmes structures hiérarchiques (éducation nationale et association). Enfin, il existe de nombreux liens organisés par le coordonnateur du CSA, notamment pour rendre viables le dispositif et l'articulation quotidienne des

14 Le Centre national des ressources textuelles et lexicales (https://www.cnrtl.fr/definition/fonction ) définit notamment le mot « fonction » comme le « rôle propre joué par un élément au sein d'un ensemble dont les parties sont solidaires ». Nous adopterons donc ici cette définition.

15 https://www.cnrtl.fr/definition/fonction

16 Ce que nous avons défini comme étant l'identité du dispositif (Bourdon, Toullec-Théry, 2016) 


\section{$\&$}

\section{REVUE HYBRIDE DE L'ÉDUCATION}

activités de l'élève (cours, accompagnements, soins). Il existe en effet des relations entre les acteurs qui sont pédago-didactiques, hiérarchiques, de régulations, d'accompagnement, voire politiques, pour ce qui est de la mise en œuvre du système et son financement, comme le synopsis ci-après le montre.

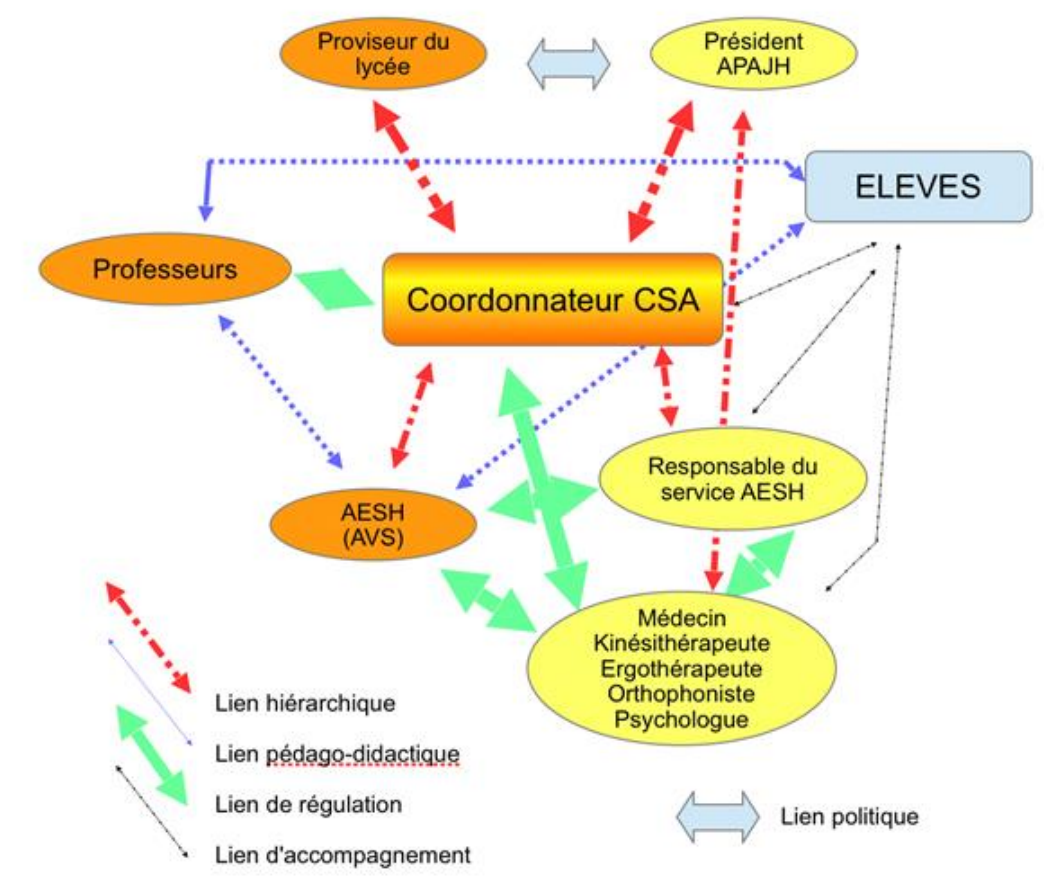

Figure 3. Modélisation des liens fonctionnels

Nous pouvons alors mesurer la complexité du système avec cette première représentation des liens entre les acteurs. II s'agit alors maintenant de mieux identifier comment le système fonctionne et vient en appui de la scolarisation à travers les différentes fonctions ressources mises en œuvre.

\section{La fonction information pour les acteurs du lycée : la fiche de "besoins élèves "}

En début d'année scolaire, le coordonnateur du CSA établit avec l'élève une fiche de besoins qui permet d'identifier, d'une part, les difficultés potentiellement rencontrées par l'élève en situation scolaire, d'autre part, proposer des aménagements ou fonctionnements spécifiques pour favoriser l'accessibilité. Celle-ci est mise à la disposition de tous les personnels qui sont en lien avec l'élève (professeurs en premier lieu, 


\section{$\&$}

\section{REVUE HYBRIDE DE L'ÉDUCATION}

$\mathrm{AESH}$, membres de la direction, personnels de service si nécessaire, surveillants d'internat le cas échéant). Elle est simple, descriptive, établie par l'intéressé.e lui.elle-même et peut se compléter individuellement par chaque acteur selon ses observations, au gré des rencontres dans le courant de l'année avec l'élève. Elle participe à l'accessibilisation telle que définie par Benoit et Feuilladieu (2017) à propos des outils ${ }^{17}$.

Nous sommes confrontés ici à des lycéens en mesure d'énoncer leurs besoins : "Nous, on se base, non sur les besoins des profs, mais sur les besoins des jeunes », souligne une éducatrice.

Dès que les besoins des élèves et de l'accessibilité aux situations sont abordés avec les professeurs, c'est de cette fiche dont ils parlent spontanément: déjà en adaptation de documents... ça, dès le début de l'année, on peut s'y référer parce que justement, on sait avec la fiche de présentation, s'il faut agrandir, s'il faut des choses comme ça. Après, il y a des choses plus précises, en termes de polices de caractère, d'organisation des documents sur une ou deux colonnes, des choses comme ça, mais ça, c'est pas toujours précisé nettement. Certains élèves, en plus, peuvent avoir une évolution là aussi, soit de leurs besoins, soit de leur volonté, de leurs choix... Moi, je leur demande systématiquement, je leur présente toujours d'abord un document, je leur présente un document $A 3$ et puis je leur demande... je lui demande, est-ce que tu préfères en $A 3$ ou $A 4$, puis si c'est en $A 3$, comment tu le préfères, est-ce que tu le préfères en paysage, voilà. Après la police de caractère, qu'est-ce que tu préfères, voilà on voit ça au fur et à mesure, et on le travaille au fur et à mesure (Professeur P1).

$\mathrm{P} 1$ organise donc les compensations selon deux modes :

en amont, par rapport à des obstacles potentiels dans sa discipline (ici, l'histoire) ;

- $\quad$ par rapport à ce que précise la fiche d'informations qui émane du CSA.

Il ajuste également sa séance aussi in situ au fil de ses observations ou des demandes de l'élève. Si le professeur mentionne la fiche de présentation d'emblée dans son discours, c'est qu'elle est un outil central dont il se saisit, mais cette fiche semble être prise parfois au pied

17 Outils numériques en ce qui concerne cet article, mais que l'on peut aussi exporter vers d'autres types d'outils. 


\section{8}

\section{REVUE HYBRIDE DE L'ÉDUCATION}

de la lettre. Elle revêt alors un aspect prescriptif : « il faut agrandir », dit-il à deux reprises. Pour autant, la mise au point des compensations se fait aussi en lien avec l'élève : «je leur demande, qu'est-ce que tu préfères ? On le travaille au fur et à mesure ».

Dans quel cadre et à quelles fins est proposée cette fiche? Le jour de la prérentrée, si on a réussi à bien faire notre boulot dans ce dispositif favorisant l'inclusion, on va être susceptible, on va être en mesure de donner aux enseignants, ce qu'on appelle une fiche de présentation [...] qui va donner des éléments sur la situation du futur élève qu'il aura dans sa classe le lendemain, qui ne sera pas un descriptif de la situation de handicap ou des situations de handicap du jeune, mais ce que nous aura dit le jeune sur les conséquences, que lui voit, au niveau scolaire, de ses situations de handicap (Coordonnateur du CSA).

Cette fiche, rédigée en amont de la rentrée à partir de ce qu'exprime l'adolescent sur ses besoins, est pensée comme un guide à l'intention des professeurs, principalement. Le coordonnateur y accorde une haute importance puisque "bien faire son boulot ", c'est diffuser cette fiche à temps. Elle est ainsi diffusée auprès des professionnels en format papier, automatiquement. Pourtant, cette fiche prend un statut paradoxal. En effet, si elle est une aide (avec des indicateurs précis), elle peut empêcher d'innover, en prenant un statut prescriptif : ce qui est inscrit devient alors ce qu'il faut faire. Cette fiche qui donne aussi des éléments sur les attributions de l'AVS peut, de plus, le reléguer à des tâches subalternes. II y a donc un risque malgré l'intérêt souligné par tous les acteurs, d'une injonction à faire comme la fiche l'indique, son élaboration et son usage requièrent alors un cadrage pour éviter la prescription.

\section{La fonction interface liée aux missions du coordonnateur du CSA et de l'encadrement du lycée}

Nous avons longuement détaillé cette fonction dans les deux articles référencés au début du présent texte (Bourdon et Toullec-Théry, 2016, 2018). Pour autant, nous souhaitons y revenir, car cette fonction d'interfaçage est évoquée tant par les professeurs que le coordonnateur du CSA ou les cadres dirigeants du lycée et de l'association médico-sociale.

En effet, la fonction du coordonnateur du CSA s'exerce principalement, comme son nom le suggère, au carrefour des partenariats, mais aussi des activités de l'élève. II s'agit donc, comme il le précise avec cet exemple, de permettre la scolarisation la plus ordinaire possible en tenant compte des besoins des élèves et des situations des professionnels : l'objectif, quand un 


\section{$\&$}

\section{REVUE HYBRIDE DE L'ÉDUCATION}

élève $a$, à la fois son projet d'être élève dans un lycée ordinaire, en inclusion, mais que, parallèlement, il y a une prise en charge médico-sociale, on a souvent tout à gagner de mettre en lien par exemple l'orthophoniste qui assure la prise en charge et le prof de français, par exemple de la classe, qui doit amener l'élève à la fin de son année, pour les épreuves de bac anticipé de français, à tel niveau d'exigence. Ça veut pas dire qu'en les faisant se rencontrer, on va demander à l'orthophoniste de devenir prof de français bis, et vice et versa, mais ça va créer des synergies, je dirais, entre deux cultures professionnelles qui sont distinctes, mais qui ont finalement les mêmes objectifs de base qui sont, l'accompagnement global du jeune dans ses besoins particuliers et qu'il avance dans ses apprentissages scolaires.

Nous savons que ces rencontres sont gourmandes en temps de travail et sont un frein parfois à la mise en œuvre du projet de scolarisation inclusive. II s'agit alors d'identifier dans ce système ce qui permet la scolarisation sans surcharge de travail au-delà des aspects pédago-didactiques. Le proviseur du lycée conforte cette position en indiquant: je crois que c'est lié à la mutualisation tout simplement, c'est-àdire que là il y a une optimisation des ressources qu'on n'a pas dans un établissement où c'est plus individuel, on a un travail de coordination qui est fort, qui permet d'optimiser [...] Par exemple, j'ai l'impression que les professeurs ne sont pas tenus obligatoirement en dehors des réunions habituelles de conseil de classe ou de concertation, à travailler plus, ou à rester plus ou aller nécessairement voir les gens du centre de scolarité adaptée, etc. II y a quelque chose qui circule qui leur permettent de travailler en complémentarité parce que le coordonnateur ou moi-même assurons une certaine cohésion.

La complexité des situations éducatives, la gestion souvent difficile des contraintes liées aux emplois du temps variés de ces élèves, à la singularité des interventions, demandent aux professionnels comme aux élèves de s'adapter dans un fonctionnement proche de la haute couture, c'est-à-dire " cousu main ». La fonction d'interface, lorsqu'elle est identifiée comme un outil d'appui et efficace, permet de mettre du lien, du liant, de la cohérence, de la cohésion autour, non pas de l'élève à BEP, mais de ses activités tant scolaires, rééducationnelles que sociales. 


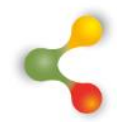

\section{REVUE HYBRIDE DE L'ÉDUCATION}

II s'agit donc d'une fonction essentielle qu'il est nécessaire de penser, comme le souligne ce professeur : l'interface, c'est la zone de contact. Donc cette zone de contact ça peut être entre deux espaces ou deux personnes qui sont opposées, pour créer de l'échange. Pour moi, à mon sens, l'idée d'interface, elle est là, c'est-à-dire créer de l'échange, créer des échanges, le coordonnateur du CSA est une interface parce que c'est lui qui crée l'échange, qui facilite l'échange entre les profs, les élèves, les AVS, les familles, donc là il est interface à tous les niveaux.

\section{La fonction adaptation dans le lien pédagogique}

Cette fonction qui s'inscrit plus particulièrement dans la relation pédagogique, telle que nous l'identifions, est primordiale; c'est avec elle que les professeurs pourront ajuster leurs enseignements, leurs étayages, s'engager dans une analyse didactique pour donner accès aux connaissances et compétences. Elle s'exerce principalement entre les professeurs et les élèves, mais aussi entre professeurs et AESH. C'est ce triangle relationnel et d'activités, dans lequel s'inscrit l'adaptation aux besoins. Pour autant, elle nécessite des clarifications, car comme le soulignent ces extraits d'entretiens, la relation entre ces trois parties n'est pas de fait. On trouve trace de cette dissymétrie relationnelle, surtout dans les discours d'AVS interviewés, comme ici : « lls ont leur rôle d'enseignant et ils s'adressent à la classe. Moi, je fais partie un peu du décor parfois ». Dans ce cas, la présence de l'AVS, comme l'indique ce professeur, module peu ses manières de faire usuelles : «Bon, surtout en début d'année, on échangeait s'il y avait des choses à caler, non y a pas eu beaucoup de modifications [de mes pratiques]» (P2). Sur les trois professeurs interviewés, deux ne savent ainsi pas que les AVS ont une "spécialité " disciplinaire «quel est leur type de formation, je ne sais pas, mais en tout cas, ils n'ont pas une spécialité disciplinaire particulière » (P3). Alors qu'ils sont effectivement recrutés selon leurs compétences pour intervenir au plus près des besoins, un AESH spécialiste des "maths", un autre "littérature » ou encore "histoire ou géographie », "langue », etc. La mutualisation permettant d'accompagner l'élève dans les disciplines selon ses besoins.

Les élèves perçoivent alors ces difficultés liées au contexte professionnel professeur-AESH : «Y a certains profs qui vont vraiment essayer de s'adresser qu'à nous et l'AVS, elle sera transparente et d'autres qui vont directement parler à l'AVS comme si on n'était pas là. $Y$ a les deux extrêmes » (Élève de $1^{\text {re }}$ scientifique).

$\mathrm{Si}$, selon les professeurs, l'AESH détient une place fluctuante en classe (voire subalterne parfois), ce n'est pas le cas dans les autres institutions dont il fait partie. II en est, en effet, un pilier : il est membre à 


\section{$\&$}

\section{REVUE HYBRIDE DE L'ÉDUCATION}

part entière des Équipes de Suivi de Scolarisation (ESS) qui se réunissent pour chaque élève une fois par an, sans compter sur d'autres réunions au cours de l'année.

Cette fonction « adaptation » va donc s'installer au fil des discussions, généralement informelles, entre professeurs et AESH ou professeurs et élèves. Elle va aussi s'appuyer sur la fiche de présentation qu'ils complètent au fil de l'année scolaire pour ajuster au mieux leurs enseignements. Moi j'ai besoin énormément de parler soit avec les AVS, j'ai aussi besoin de consulter la fiche de début d'année et surtout presque à chaque cours, si nécessaire bien sûr, je discute avec les élèves. Cela me permet de voir ce que je fais avec eux et d'ajuster pour le cours prochain, ou même dans l'instant (Professeur 3).

Les adaptations pédagogiques sont de natures variées, il peut s'agir « tout simplement " d'un agrandissement ou d'un choix de police de caractères, jusqu'à la mise à disposition de situations didactiques particulières pour répondre aux besoins des élèves.

Cette fonction «adaptation" va ainsi favoriser la scolarisation inclusive en ce sens qu'elle donne accès aux élèves aux savoirs de tous en prenant en compte les besoins de chacun. II s'agit alors pour les professeurs, d'identifier les ressources dont ils ont besoin autant dans l'ici et maintenant du cours, que dans les temps de préparation préalable. Elle permet effectivement aux élèves et aux professionnels (notamment les professeurs) d'ajuster au mieux les cours aux besoins.

\section{La fonction accessibilité permet à l'élève d'accéder pleinement à ses activités}

Comme nous l'avons vu, si la fonction "adaptation" est directement liée au système pédagogique et didactique, la fonction " accessibilité » est plus transversale et s'inscrit dans un processus global qui, comme le souligne Serge Ébersold (2020) « [l'école inclusive] illustre l'avènement d'une école post-disciplinaire, consécutif à l'avènement d'une société de la connaissance faisant de l'éducation un vecteur de protection sociale et de l'accessibilité des environnements scolaires un impératif ». L'accessibilité dépasse donc les procédures et ne se réduit pas à aux conséquences des compensations. "L'implication des intéressés dans les processus à l'œuvre permet de lier l'effet capacitant des pratiques aux subjectivités individuelles et au sens pratique entourant les usages des lieux, des objets, des services" (Ébersold, 2019). Ainsi, compte tenu de l'âge des élèves et du caractère souvent inné des troubles, ils ont une assez bonne connaissance de leurs fonctionnements et besoins. C'est aussi un public qui a depuis longtemps bénéficié de rééducation, de soins 


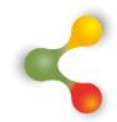

\section{REVUE HYBRIDE DE L'ÉDUCATION}

médicaux, qui entretient avec le corps médical ou de rééducation une relation de longue haleine depuis l'enfance. Dans le paradigme de la $\mathrm{CIF}^{18}$, l'activité des personnes en situation de handicap est placée au centre du système et a des effets sur la personne et ses fonctions anatomiques. Le contexte dans lequel ces activités se déroulent agit aussi fortement sur la façon dont elles peuvent se développer. Ainsi rendre les savoirs et les relations sociales accessibles est un enjeu fort d'inclusion. Si nous identifions la fonction "accessibilité » comme une ressource au sein de l'école, c'est qu'il ne s'agit pas de penser seulement la compensation des troubles, mais l'identification des besoins, des freins et empêchements contextuels pour accéder aux savoirs et aux interactions entre pairs. Dans l'analyse des interactions dans le système du Lycée Les Bourdonnières, nous avons principalement observé le lien entre les $A E S H$, les professionnels de santé ou de rééducation et les élèves en lien avec les professeurs. C'est dans ce triangle soignants-élèves-professeurs qu'il s'origine et se maintient.

Comme en témoigne le psychologue de l'établissement,

on a fait des formations en interne aux profs pendant des années aussi pour leur expliquer, ou à chaque fois qu'il y a des profs nouveaux qui viennent, pour chaque type de handicap, les conséquences que ça a au niveau des apprentissages, des troubles cognitifs, des grands IMC tout ça, c'est pas seulement le fauteuil qui est leur handicap, c'est les difficultés logico maths et y'a des fonctions qui ont été aussi atteintes dans l'atteinte motrice cérébrale du départ. II y a des jeunes qui sont des nullités en maths pendant toutes leurs études et puis c'est quasiment impossible d'y remédier et ils vont arriver à contourner les difficultés. II y a aussi ce témoignage de la coordonnatrice des $A E S H$ qui indique : on leur donne déjà tout un protocole et on leur parle du travail, des jeunes, de leur handicap, des compensations... Enfin, on leur parle pas forcément de leur handicap, de l'handicap même, parce que ça c'est pas très important. Le plus important, c'est les compensations, c'est les conséquences du handicap sur les apprentissages : donc qu'est-ce qu'on doit mettre en place pour que les jeunes aient la même égalité de chances que les autres... au niveau du scolaire. Donc là, au début du

18 Classification internationale du fonctionnement de la santé et du handicap https://www.ehesp.fr/international/partenariats-et-reseaux/centre-collaborateuroms/classification-internationale-du-fonctionnement/ 


\section{$<$}

\section{REVUE HYBRIDE DE L'ÉDUCATION}

mois de septembre, avec les nouveaux, je fais... la kiné et moi-même, on fait une formation sur les gestes et postures et je les suis à peu près sur trois semaines pour leur montrer comment on s'occupe d'un jeune à besoins particuliers.

Enfin, nous retenons les propos d'un membre de l'équipe dirigeante de l'APAJH qui indique je pense que le fait d'avoir une équipe mutualisée avec des compétences diverses ça permet peut-être aussi, non seulement de remplir les plages horaires nécessaires, mais peut-être aussi d'adapter l'intervention de l'AVS en fonction du cours qui va être assuré. C'est aussi un moyen pour que chacun puisse apporter son soutien selon les besoins de l'élève et dans son champ de compétences. Adapter aux besoins de l'élève et aux exigences du prof ou de la discipline est quand même compliqué... mais c'est primordial.

La fonction "accessibilité » permet effectivement aux élèves de participer tant d'un point de vue social que dans la réalisation et l'inscription dans le métier d'élève. II serait réducteur de considérer que cette fonction est uniquement circonscrite dans l'accès aux locaux scolaires et collectifs. C'est grâce à cette fonction qui favorise l'engagement que les élèves à BEP peuvent s'inscrire dans un contexte avec ses normes, ses routines, ce qui leur permet de s'envisager comme "membres » de la communauté qu'ils fréquentent. L'expérience individuelle rendue accessible en milieu régulier, est une constituante de la reconnaissance de tous à bénéficier du droit à accéder à ses choix, appuyer par les organisations locales (ici l'ensemble du dispositif dont le CSA est emblématique) pour garantir le droit à être élève, sujet, acteur dans ce lycée.

\section{La fonction régulation pour l'articulation du projet autour de l'élève}

Cette dernière fonction proposée va s'inscrire dans des systèmes de coordination, d'organisation à travers un certain nombre de réunions, notamment. Nous savons que les enseignants qui participent à des projets d'inclusion scolaire disent que les temps de réunion sont précieux, mais très chronophages et représentent un frein à un plus grand engagement (Bourdon, 2020). Il s'agit donc de regarder comment les acteurs s'inscrivent dans des régulations formelles et informelles et comment celles-ci permettent de constituer des ressources pour l'inclusion. Dans le cas de projet de scolarisation inclusive, de multiples acteurs interviennent dans le champ scolaire bien que certains relèvent d'autres domaines professionnels, je pense notamment aux professions paramédicales, aux psychologues, médecins ou éducateurs. Par ailleurs, il existe en France, comme dans beaucoup de pays, des organisations institutionnalisées, 


\section{$\&$}

\section{REVUE HYBRIDE DE L'ÉDUCATION}

notamment les réunions qui relèvent du droit et de l'obligation faites aux parties de se réunir pour décider de l'orientation. C'est le cas pour ce qui concerne la réunion de l'équipe de suivi de la scolarisation (ESS) qui a été instituée par la loi de 2005 sur le handicap ${ }^{19}$, ou du conseil de classe dont nous avons déjà parlé qui est une obligation pour tous les établissements scolaires du $2^{\mathrm{d}}$ degré. II existe par ailleurs des réunions internes aux équipes qui relèvent de l'usage (réunion de synthèse hebdomadaire ou mensuelle, par exemple).

La coordonnatrice des AESH souligne aussi les relations informelles avec les professeurs de façon à réguler la scolarité des élèves à $\mathrm{BEP}$ : les profs de toute façon savent qu'il y a le centre de scolarité adaptée, savent qu'ils sont accompagnés, que tel jeune est accompagné par le centre de scolarité adaptée, donc bien sûr ils peuvent nous contacter, soit par téléphone ou venir directement nous voir.... Ils viennent souvent nous rencontrer.

Le proviseur du lycée insiste sur la nécessité de coordination au niveau des instances de décision :

moi, je vois, les partenaires, je vois les représentants de l'APAJH, on les voit, pas assez régulièrement, on les voit une fois par an. On se pose pour faire le point sur le fonctionnement. Bon, je souhaiterais que ça soit plus régulier, justement, c'est l'objet de ma discussion tout à l'heure d'avoir un point plus régulier sur le fonctionnement, pour faire des synthèses.

Ce travail de régulation à plusieurs niveaux, au local et en contexte dans l'ici et maintenant, lors de réunions régulières programmées au niveau des instances décisionnelles permettent de fluidifier le système, à la fois pour régler des questions précises liées à tel ou tel élève à BEP, et aussi pour favoriser l'harmonisation du dispositif et sa régulation.

Alors, il y a une réunion une fois tous les 15 jours avec le directeur, avec les AVS. Et puis ben... enfin moi je suis toute la journée là, donc euh... on se voit régulièrement, on a des réunions informelles en fait, on se parle tout le temps... précise encore la coordonnatrice des AESH.

19 http://www. mdph.fr/index.php?option=com content\&view=article\&id=105:la-loi-du-11fevrier-2005 


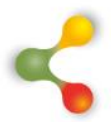

\section{REVUE HYBRIDE DE L'ÉDUCATION}

Le coordonnateur du centre scolaire adapté (CSA) évoque la complexité des rapports et des cultures entre l'éducation nationale et le médico-social. II dit aussi la complexité des interventions et des dispositifs (transport en taxi pour les élèves, instances de décisions, services de soins, scolarité et aménagement des cours...), autant de moments et d'instances qui demandent une régulation autour du projet de l'élève,il y a plein d'interlocuteurs dont, enfin, dont on n'a même pas parlé. Alors je peux en citer quelques-uns: la $\mathrm{MDPH}^{20}$, les services transports des conseils généraux pour le taxi des élèves en situation de handicap, le conseil régional pour l'aménagement des locaux puisque la collectivité territoriale de rattachement des lycées c'est la région, et puis... et puis j'en passe plein. Enfin j'ai parlé uniquement, enfin, surtout, des enseignants, sur le lycée. J'aurais pu évoquer les services de l'intendance, par exemple la mise en place des livres numériques, ça, ça passe par l'intendance.

Nous mesurons combien la régulation des différents acteurs, professionnels ou instances est utile pour fluidifier le parcours scolaire et éviter les ruptures, permettre des transitions les mieux anticipées possible. La complexité et la multiplicité des interlocuteurs pour répondre aux besoins des élèves et des professionnels pourraient, si la coordination n'est pas efficiente, amener à un dysfonctionnement du système. La fonction de régulation est semble-t-il essentiellement pour que chacun puisse s'y appuyer et trouver des ressources.

\section{Synthèse}

Nous avons pu identifier cinq fonctions ressources qui se mettent en œuvre dans cet établissement pour accompagner la scolarisation inclusive des élèves à besoins éducatifs particuliers :

- fonction informations : besoins des élèves/professionnels ;

- fonction adaptation : aspects pédago-didactiques ;

- fonction accessibilité : ajustement aux besoins des élèves pour l'accès aux savoirs, au bâti et aux pairs.es ;

- fonction interface: permet de faire le lien entre les instances et les acteurs ;

- fonction régulation : favorise l'ajustement et le suivi du parcours scolaire.

20 «Maison départementale des personnes handicapées » qui régulent les questions autour de l'insertion et du handicap, délivre le statut de personne handicapée. II en existe une par département (territoire géographique). 


\section{REVUE HYBRIDE DE L'ÉDUCATION}

Nous pouvons alors observer avec ce synopsis comment ces fonctions agissent, à quel niveau, avec quels acteurs.

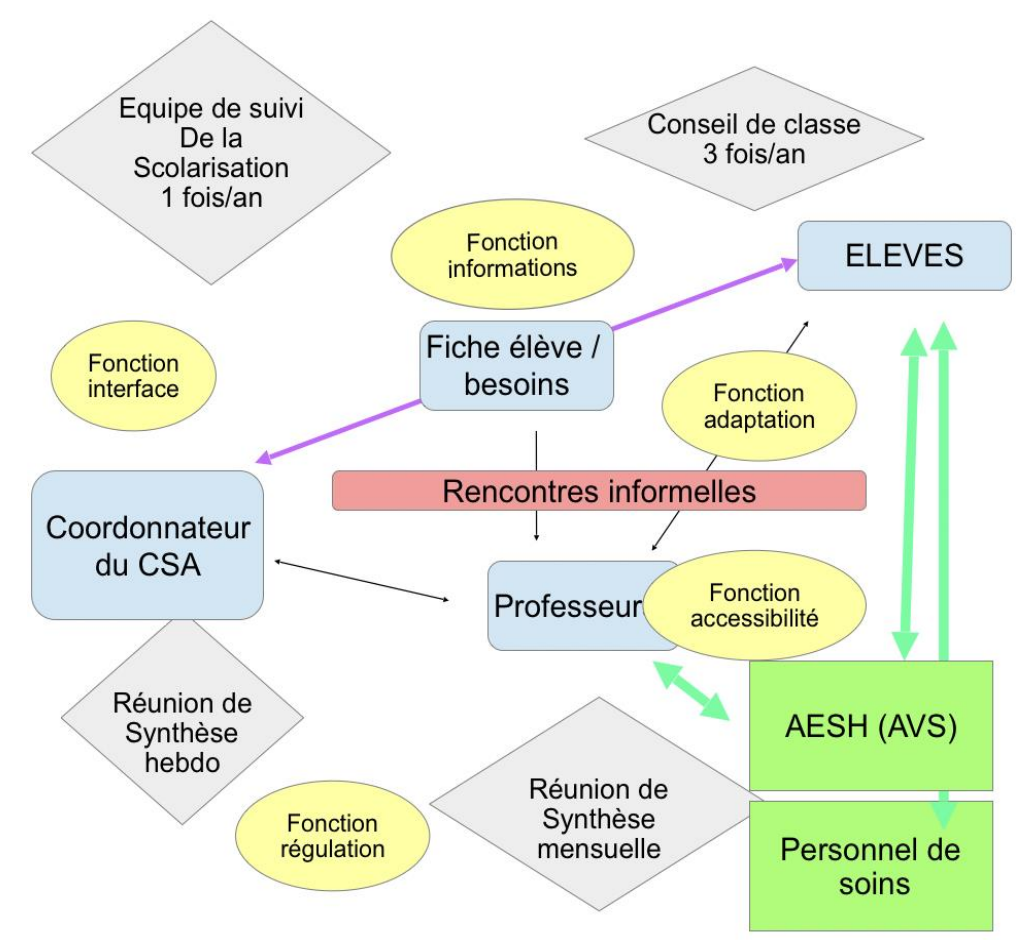

Figure 4 : Modélisation générale du système inclusif du Lycée Les Bourdonnières

La complexité des organisations, la multitude de professionnels présents ou non au quotidien (certains médecins n'interviennent qu'une fois par an), les cultures différentes entre l'éducation nationale et les services médico-sociaux, les pratiques professionnelles variées... nécessitent que chacun puisse trouver des ressources au-delà de la fonction ressource d'un enseignant dédié à celle-ci. Y compris lorsqu'un enseignant est missionné pour assurer ce travail, comme c'est le cas du coordonnateur du CSA ou de la coordonnatrice des AESH, le proviseur du lycée... il est indéniable, nous l'avons vu, qu'un certain nombre de ressources sont saisies par les acteurs sur des modes tant formels qu'informels. C'est la complexité des systèmes qui rend nécessaire la clarification des ressources pour y accéder selon les besoins de chacun et les périodes de l'année.

C'est à ce prix que le parcours de scolarisation inclusive favorisera les transitions les plus fluides. 


\section{REVUE HYBRIDE DE L'ÉDUCATION}

\section{Références}

Bautier, E. (1995). Pratiques langagières, pratiques sociales. L'Harmattan.

Benoit, H. et Feuilladieu, S. (2017). De la typologie des outils numériques dans le champ des EIAH à leur opérationnalité inclusive. La nouvelle revue de l'adaptation et de la scolarisation, 78(2), 25-45.

Blanchet, A. et Gotman, A. (1992). L'enquête et ses méthodes : l'entretien. Nathan, collection 128.

Bourdon, P. (2007). Les enfants en situation de handicap, comment se construisent-ils avec et dans l'école ?. Médecine Thérapeutique Pédiatrie, 10(4), 253-259. DOI : 10.1684/mtp.2007.0116

Bourdon, P. (2008). De l'enseignant spécialisé à l'enseignant ressources, Les Cahiers pédagogiques, 459, janv., 27-28

Bourdon, P (2016). Parcours scolaire et scolarisation inclusive chez les élèves avec un trouble moteur. Dans Jacques, M.-H. (dir). Transitions en contexte scolaire (p.379-390). Presses de l'Université de Rennes.

Bourdon, P. (2018). School Career and Inclusive School between Breaks and Continuities in Children and Adolescents Suffering from a Disabling Disease. Revista Romaneasca pentru Educatie Multidimensionala, 10(4), 112-118.

Bourdon, P. (2019). Continuités et ruptures dans le parcours en ULIS pro. Les Cahiers pédagogiques. Coll. hors-série numériques.

Bourdon, P. (2020). Le parcours scolaire comme indicateur de scolarisation inclusive : le cas des Ulis pro [document accepté pour publication]. Ressources : Université de Nantes.

Bourdon, P. et Toullec-Thery, M. (2016). Analyse des effets du dispositif de scolarisation inclusive au lycée polyvalent Les Bourdonnières à Nantes. La nouvelle revue de l'adaptation et de la scolarisation, (2), 181-200.

Bourdon, P. et Toullec-Théry, M. (2018). Équipe pluridisciplinaire en appui à la scolarisation inclusive : le cas du Lycée Les Bourdonnières à Nantes (France). Revue Suisse de pédagogie spécialisée, 18-25.

Clot, Y. (1995). L'échange avec un sosie pour penser l'expérience. Société française, (53), 51-55.

Ébersold, S. (2020). École inclusive, société de la connaissance et impératif d'accessibilité. Carnets rouges, janvier (18), 7-9. http://reseau-ecole.pcf.fr/114315

Ébersold, S. (2019). L'accessibilité ou la solidarité reconfigurée? Vie sociale, 27(3), 93-108. doi:10.3917/vsoc.193.0093. 


\section{REVUE HYBRIDE DE L'ÉDUCATION}

Leontiev, A. (1975). Activité, conscience, personnalité (traduit, 1984). Éditions du progrès.

Passeron, J.-C. et Revel, J. (2005). Penser par cas. Éditions de l'EHESS.

Rochex, J.Y. (1995). Le sens de l'expérience scolaire. PUF

Rosnay (de), J. (1975). Le macrosocope : vers une vision globale. Seuil

Sensevy G. et Mercier A. (2007). Agir ensemble: l'action didactique conjointe du professeur et des élèves. Presses de l'Université de Rennes.

Toullec-Théry, M. (2013). Mises en œuvre parfois paradoxales dans l'action conjointe enseignant-AVS-élève handicapé. Deux études de cas à l'école primaire. Dans T. Assude et J.-M. Pérez (dir.) Pratiques inclusives et savoirs scolaires (p.125-142). Presses Universitaires de Nancy.

Tremblay, P. (2015). Le coenseignement en primaire : rôles respectifs des enseignants ordinaires et spécialisés. La nouvelle revue de l'adaptation et de la scolarisation, (2), 107-119. 